Research, Society and Development, v. 9, n. 8, e688986058, 2020

(CC BY 4.0) | ISSN 2525-3409 | DOI: http://dx.doi.org/10.33448/rsd-v9i8.6058

Diagnósticos de enfermagem em recém-nascidos de uma maternidade do interior do

\title{
Nordeste brasileiro
}

Nursing diagnoses in newborns in a maternity in a Northeast brazilian city

Diagnósticos de enfermería en recién nacidos en una maternidad del interior del Noreste del Brasil

Recebido: 26/06/2020 | Revisado: 29/06/2020 | Aceito: 10/07/2020 | Publicado: 20/07/2020

Fatumata Binta Embalo

ORCID: https://orcid.org/0000-0003-4594-5166

Universidade da Integração Internacional da Lusofonia Afro-Brasileira, Brasil

E-mail: fatumata15@hotmail.com

Emília Soares Chaves Rouberte

ORCID: https://orcid.org/0000-0001-9758-7853

Universidade da Integração Internacional da Lusofonia Afro-Brasileira, Brasil

E-mail: emilia@unilab.edu.br

Isaura Leticia Tavares Palmeira Rolim

ORCID: https://orcid.org/0000-0002-8453-2543

Universidade Federal do Maranhão, Brasil

E-mail:leticiaprolim@yahoo.com.br

Rafaella Pessoa Moreira

ORCID: https://orcid.org/0000-0003-2341-7936

Universidade da Integração Internacional da Lusofonia Afro-Brasileira, Brasil

E-mail: rafaellapessoa@unilab.edu.br

Maria Isis Feire de Aguiar

ORCID: https://orcid.org/0000-0002-6068-1747

Universidade Federal do Ceará, Brasil

E-mail:isis_aguiar@yahoo.com.br

Débora Oliveira Pordeus

ORCID: https://orcid.org/0000-0003-0518-2969

Universidade Federal do Maranhão, Brasil

E-mail: debbyoliver@yahoo.com.br

Jocelha Maria Costa Almeida

ORCID: https://orcid.org/0000-0002-3147-954X 
Hospital Municipal Djalma Marques, Brasil

E-mail: jocelhacosta@gmail.com

\title{
Resumo
}

Objetivo: Identificar a prevalência de diagnósticos de enfermagem e suas características definidoras em recém-nascidos, com base nos indicadores clínicos das respostas humanas identificadas. Metodologia: Foi realizado um estudo descritivo e transversal com 45 recémnascidos no período de dezembro de 2018 a fevereiro de 2019, com dados coletados por meio de exame físico e levantamento do histórico de enfermagem. Para a nomeação dos diagnósticos, foi utilizada a Taxonomia II da NANDA-I. Resultados: Foram identificados os seguintes diagnósticos: Risco de infecção (80\%), Risco de desenvolvimento atrasado (55.5\%), Amamentação ineficaz (28.8\%), Padrão respiratório ineficaz (17.7\%), Risco de débito cardíaco diminuído (4.4\%). Considerações finais: A investigação revelou cinco diagnósticos de enfermagem com suas características definidoras, fatores relacionados ou fatores de risco em recém-nascidos. Os diagnósticos identificados na maior parte dos recém-nascidos relacionavam-se ao risco de infecção e risco no atraso de desenvolvimento. Ressaltando deste modo, a importância do pensamento crítico e reflexivo, baseado em evidências científicas por parte do enfermeiro para assegurar uma assistência qualificada por meio do uso da sistematização de assistência de enfermagem.

Palavras-chaves: Diagnóstico de enfermagem; Recém-nascido; Classificação; Cuidados de enfermagem; Enfermagem.

\begin{abstract}
Objective: To identify the prevalence of nursing diagnoses and their defining characteristics in newborns based on clinical indicators of human responses identified. Methodology: A descriptive and cross-sectional study was carried out with 45 newborns in the period from December 2018 to February 2019, with data collected by means of physical examination and survey of the nursing history. For the appointment of diagnoses, the NANDA-I Taxonomy II was used. Results: The following diagnoses were identified: Risk of infection (80\%), Risk of delayed development (55.5\%), Ineffective breastfeeding (28.8\%), Ineffective respiratory pattern $(17.7 \%)$, Risk of decreased cardiac output (4.4\%). Final thoughts: The research revealed five nursing diagnoses with their defining characteristics and related factors or risk factors in newborns. The identified diagnoses in most of the newborns were related to the risk of infection and risk of delayed development. In this way, the importance of critical and
\end{abstract}


reflective thinking is emphasized, based on scientific evidence on the part of the nurse to ensure a qualified assistance using the systematization of nursing assistance.

Key words: Nursing diagnosis; Newborn; Classification; Nursing care; Nursing.

\section{Resumen}

Objetivo: Identificar la prevalencia de los diagnósticos de enfermería y sus características definitorias en recién nacidos, basándose en los indicadores clínicos de respuestas humanas identificadas. Metodología: Se realizó un estudio descriptivo y transversal con 45 recién nacidos en el período comprendido de diciembre de 2018 a febrero de 2019, con datos recolectados por medio de un examen físico y estudio del historial de enfermería. Para el nombramiento de los diagnósticos, se utilizó la Taxonomía II de la NANDA-I. Resultados: Se identificaron los siguientes diagnósticos: Riesgo de infección (80\%), Riesgo de retraso en el desarrollo (55,5\%), Lactancia materna ineficaz (28.8\%), Patrón respiratorio ineficaz (17,7\%), Riesgo de gasto cardiaco disminuido $(4,4 \%)$. Consideraciones finales: La investigación reveló cinco diagnósticos de enfermería con sus características definitorias y factores relacionados o factores de riesgo en los recién nacidos. Los diagnósticos identificados, en la mayoría de los recién nacidos, estaban relacionadas con el riesgo de infección y el riesgo de retraso en el desarrollo. De esta manera, se destaca la importancia del pensamiento reflexivo y crítico, basado en la evidencia científica por parte de la enfermera para garantizar una asistencia calificada a través de la utilización de la sistematización de la asistencia a la enfermería.

Palabras clave: Diagnóstico de enfermería; Recién nacido; Clasificación; Cuidados de enfermería; Enfermería.

\section{Introdução}

O nascimento é um momento que torna a criança vulnerável, onde os cuidados assistidos antes, durante e depois do parto são determinantes na conciliação da vida saudável do recém-nascido. Esses cuidados podem diminuir a morbimortalidade neonatal, portanto, prestar assistência ao recém-nascido (RN) é uma tarefa desafiadora.

$\mathrm{O}$ enfermeiro é um dos profissionais responsáveis pelo cuidado ao $\mathrm{RN}$, cabe a ele desenvolver ações capazes de manter e promover a saúde e também prevenir os possíveis agravos. Desta forma, torna-se imperioso um cuidado especializado a fim de garantir a vida saudável fora do útero. 
Kenner (2001), relata que cerca de em $80 \%$ a $90 \%$ dos nascimentos, a adaptação do ambiente intrauterino para o extrauterino ocorre em um período rápido, de maneira fisiológica, atingindo a estabilização. Porém, alguns recém-nascidos podem apresentar intercorrências e os profissionais necessitam estar habilitados para reconhecer precocemente e intervir rapidamente nestas situações. E ainda ressalta que, em quaisquer um dos casos, há a necessidade de um cuidado de enfermagem sistematizado.

A Sistematização da Assistência em Enfermagem (SAE) trouxe uma nova forma de perfazer as ações de cuidado, e utiliza como método o Processo de Enfermagem (PE) (Oliveira, 2013).

O Processo de Enfermagem habilita o enfermeiro a planejar e executar melhor o cuidado. É constituído por etapas que são realizados em sequência para obtenção de uma assistência qualificada. Estas etapas compreendem o histórico, obtido por meio da entrevista e exame físico, o diagnóstico, o planejamento, a implementação e a avaliação. O diagnóstico de enfermagem é definido conforme Associação Norte-Americana de Diagnósticos de Enfermagem (NANDA-I) como "um julgamento clínico sobre uma resposta humana a condições de saúde/processos de vida, ou uma vulnerabilidade a tal resposta, de um indivíduo, uma família, um grupo ou uma comunidade”. Essa etapa é fundamental para o planejamento adequado das intervenções (Souza, Ferreira, Chaves, Barbosa \& Dodt, 2012 como citado em NANDA, 2010).

O diagnóstico de enfermagem (DE) é função específica do enfermeiro, ele permite a identificação dos potenciais problemas e norteia a seleção de intervenções especificas.

Leon \& Nóbrega (2012), em seu estudo sobre DE ressalta a importância de que o uso do $\mathrm{PE}$, de um modelo teórico e de um sistema de classificação promove qualidade à assistência prestada, fomentando a liberdade profissional.

Segundo NANDA-I (2010), o uso de uma linguagem padronizada traz vantagens diante da complexidade dos cuidados prestados. Nesse contexto, Elizalde \& Almeida (2006) afirmam que ao se usar uma classificação sobre DE pode oportunizar crescimento da profissão de enfermagem.

O estabelecimento dos DE melhora a organização e o delineamento do cuidado prestado, facilita a identificação de uma manifestação que mereça assistência nos níveis físicos e emocionais (Souza et al 2012 como citado em NANDA, 2010).

Dentro da complexidade do cuidado ao recém-nascido a temo, este estudo objetiva identificar os diagnósticos de enfermagem e suas características definidoras em recémnascidos de uma maternidade do interior do Estado do Ceará, Brasil. 


\section{Metodologia}

Estudo descritivo e transversal, realizado em uma maternidade do município de Redenção, interior do Estado de Ceará. Esta maternidade atende, também, outros municípios próximos. Os dados foram coletados na Unidade de Alojamento Conjunto da referida maternidade, por meio de exame físico completo dos recém-nascidos e levantamento do histórico de enfermagem. Foram realizadas visitas à maternidade durante os dias uteis do período de dezembro de 2018 a fevereiro de 2019. A amostra foi por conveniência, sendo avaliados um total de 45 recém-nascidos. Este número foi decorrente da presença dos bebês no local da de coleta de dados, no período descrito anteriormente. Como critério de inclusão: crianças recém-nascidas com idade gestacional entre 37 e 42 semanas. Foram excluídos os bebês pré-termo.

Os indicadores percepcionados das respostas humanas dos $\mathrm{RN}$ foram identificados a partir do instrumento adaptado do estudo de Monteiro (2013). Sendo utilizado as seguintes fases de Gordon (1994) para a dedução dos DE: coleta, interpretação/agrupamento das informações e nomeação das categorias.

Para a coleta de dados foi realizado exame físico do RN e levantamento do histórico de enfermagem, os dados foram interpretados pelas pesquisadoras e utilizou-se a Taxonomia II da NANDA-I (NANDA, 2018) para nomeação dos DE. A referida taxonomia é conhecida mundialmente, com tradução em mais de 20 línguas (Garcia \& Nóbrega, 2004).

Os dados da pesquisa relacionados a cada uma das avaliações foram organizados em tabelas. Primeiramente foram agrupadas as descrições das características dos recém-nascidos, e, em seguida, os dados sociodemográficas das puérperas.

Posteriormente, foram selecionadas as características definidoras, agrupados em domínios e classes da NANDA-I, 2018-2020, em seguida fatores relacionados, finalizando com os respectivos diagnósticos de enfermagem, e então, foi calculado o percentual.

A pesquisa recebeu parecer favorável, sob número de parecer 3.085.885, do Comitê de Ética em Pesquisa da Universidade da Integração Internacional da Lusofonia Afro-Brasileira - UNILAB. 


\section{Resultados}

Recém-nascidos que nasceram no período de dezembro de 2018 a março de 2019, totalizando 45 bebês. Os resultados foram tratados em função de números absolutos e relativos apresentados sob forma de tabelas, conforme mostra a Tabela 1.

Tabela 1. Distribuição dos recém-nascidos conforme características ao nascimento. Redenção, 2019.

\begin{tabular}{|c|c|c|}
\hline CARACTERÍSTICAS & $\mathbf{N}$ & $\%$ \\
\hline \multicolumn{3}{|l|}{ Sexo } \\
\hline Masculino & 20 & 44,4 \\
\hline Feminino & 25 & 55,5 \\
\hline \multicolumn{3}{|l|}{ Parto } \\
\hline Normal & 14 & 31.1 \\
\hline Cesário & 31 & 68,8 \\
\hline \multicolumn{3}{|l|}{ Idade gestacional } \\
\hline 39 semanas & 13 & 28,8 \\
\hline 40 semanas & 17 & 37,7 \\
\hline 41 semanas & 13 & 28,8 \\
\hline 42 semanas & 2 & 4,4 \\
\hline \multicolumn{3}{|l|}{ Peso ao nascer } \\
\hline$<2500 \mathrm{~g}$ & 8 & 17,7 \\
\hline $2500-3999 g$ & 34 & 75,5 \\
\hline$>3999 \mathrm{~g}$ & 3 & 6,6 \\
\hline \multicolumn{3}{|l|}{ Comprimento ao nascer } \\
\hline$<40 \mathrm{~cm}$ & 1 & 2,2 \\
\hline$\geq 40 \mathrm{~cm}$ & 26 & 57,7 \\
\hline$\geq 50 \mathrm{~cm}$ & 18 & 40 \\
\hline \multicolumn{3}{|l|}{ Aleitamento materno } \\
\hline Leite materno exclusivo & 41 & 91,1 \\
\hline Leite materno + suplementos & 4 & 8,8 \\
\hline Total & 45 & 100 \\
\hline
\end{tabular}

Fonte: Dados da pesquisa (2019). 
No que concerne a características dos recém-nascidos, verificou-se que a maioria dos recém-nascidos eram do sexo feminino (55.5\%). Quanto ao tipo de parto, 31 (68.8\%) nasceram de parto cesáreo, e 14 (31.1\%) de parto normal. A idade gestacional de 40 semanas foi a mais prevalente 17 (37.7\%). Em relação ao peso ao nascer, constatou-se uma prevalência de $\mathrm{RN}$ com peso $\geq 3000 \mathrm{~g} 34$ (75.5\%). Referente ao comprimento ao nascer, predominou o comprimento $\geq 40 \mathrm{~cm} 26(57.7 \%)$. Esta informação, assim como as demais referentes às características dos bebês foram importantes para subsidiar a identificação de diagnósticos de enfermagem.

Quanto ao valor de Apgar, todos os participantes tiveram boa vitalidade neonatal nos $1^{\circ}$ e $5^{\circ}$ minutos (Apgar $>7$ ).

No que diz respeito ao aleitamento materno, leite materno exclusivo prevaleceu com 41 $(91.1 \%)$ e leite materno mais suplementos configurou 4 (8.8\%) da amostra.

Considera-se importante apresentar também as características das mães dos bebês, pois serão elas que realizarão diretamente os cuidados a eles. Essas características estão apresentadas na Tabela 2. 
Tabela 2. Descrição das características sociodemográficas das puérperas. Redenção, 2019.

\begin{tabular}{|c|c|c|}
\hline CARACTERISTICAS & $\mathrm{N}$ & $\%$ \\
\hline \multicolumn{3}{|l|}{ Idade } \\
\hline$<16$ anos & 10 & 22,2 \\
\hline 16-34 anos & 29 & 64,4 \\
\hline$\geq 35$ anos & 06 & 13,3 \\
\hline \multicolumn{3}{|l|}{ Local de Residência } \\
\hline Redenção & 23 & 51,1 \\
\hline Outras cidades & 22 & 48,8 \\
\hline \multicolumn{3}{|l|}{ Entidade Familiar } \\
\hline Vive sem companheiro & 5 & 11,1 \\
\hline Vive com companheiro & 40 & 88,8 \\
\hline \multicolumn{3}{|l|}{ Escolaridade } \\
\hline Analfabeta & 1 & 2,2 \\
\hline Ensino fundamental incompleto & 2 & 4,4 \\
\hline Ensino fundamental & 15 & 33,3 \\
\hline Ensino médio incompleto & 2 & 4,4 \\
\hline Ensino médio completo & 18 & 40 \\
\hline Ensino superior & 7 & 15,5 \\
\hline \multicolumn{3}{|l|}{ Ocupação } \\
\hline Funcionário público & 7 & 15,5 \\
\hline Funcionário privado & 7 & 15,5 \\
\hline Estudante & 3 & 6,6 \\
\hline Do lar & 28 & 62,2 \\
\hline \multicolumn{3}{|l|}{ Renda Familiar } \\
\hline Menos de um salário & 13 & 28,8 \\
\hline Um salário & 6 & 13,3 \\
\hline Mais de um salário & 13 & 28,8 \\
\hline Não sabe & 13 & 28,8 \\
\hline Total & 45 & 100 \\
\hline
\end{tabular}

Fonte: Dados da pesquisa (2019). 
Research, Society and Development, v. 9, n. 8, e688986058, 2020

(CC BY 4.0) | ISSN 2525-3409 | DOI: http://dx.doi.org/10.33448/rsd-v9i8.6058

Considera-se que os dados sóciodemográficos das puérperas poderiam ter influência direta em características definidoras e fatores relacionados de possíveis diagnósticos de enfermagem identificados. Portanto, em relação a essas características sociodemográficas, a faixa etária predominante foi maior de 18 anos que configura 29 (64.4\%), relacionado ao local de residência, 23 (51.1\%) são de Redenção e as demais 22 (48.8\%) são de outros municípios.

Em referência à entidade familiar, a maioria das puérperas vive com companheiro numa união estável, configurando 40 (88.8\%). A escolaridade foi diversificada, predominando ensino médio completo 18 (40\%), seguido de ensino fundamental completo 15 (33.3\%), ensino superior $7(15.5 \%)$, ensino fundamental e médio incompletos tiveram a mesma prevalência de $2(4.4 \%)$ e analfabeta $1(2.2 \%)$. Em relação à ocupação, a maioria é do lar 28 (62.2\%). No que diz respeito à renda familiar, as que não souberam informar a renda familiar e as que possuem renda familiar de mais de um salário demarcaram a mesma prevalência de $13(28.8 \%)$.

Na Figura 1 estão apresentados os títulos de diagnósticos de enfermagem identificados nos recém-nascidos.

Figura 1. Prevalência dos diagnósticos de enfermagem em recém-nascidos. Redenção, 2019.

\begin{tabular}{|c|c|c|}
\hline Diagnóstico & N & $\%$ \\
\hline Risco de infecção & 36 & 80 \\
\hline Risco de desenvolvimento atrasado & 25 & 55,5 \\
\hline Amamentação ineficaz & 13 & 28,8 \\
\hline Padrão respiratório ineficaz & 8 & 17,7 \\
\hline Risco de débito cardíaco diminuído & 2 & 4,4 \\
\hline
\end{tabular}

Fonte: Dados da pesquisa (2019).

A partir dos dados apresentados na Figura 1, foram identificados no total, cinco diagnósticos de enfermagem, os quais três configuram diagnósticos com foco no problema e dois diagnósticos de risco. Os diagnósticos com foco no problema referem-se aquelas respostas atuais e vigentes nos recém-nascidos $(\mathrm{RN})$ e os diagnósticos de risco referem-se a condições em que os $\mathbf{R N}$ ameaçam de desenvolver uma resposta não beneficiada.

Assim, o DE mais prevalente foi Risco de infecção identificada em 35 (77.7\%) dos casos, seguido de Risco de desenvolvimento atrasado 25 (55.5\%), Amamentação ineficaz 13 (28.8\%), Padrão respiratório 8 (17.7\%) e Débito cardíaco diminuído 2 (4.4\%). 
Research, Society and Development, v. 9, n. 8, e688986058, 2020

(CC BY 4.0) | ISSN 2525-3409 | DOI: http://dx.doi.org/10.33448/rsd-v9i8.6058

Na Figura 2 apresenta-se a distribuição da frequência das características definidoras e fatores relacionados segundo os diagnósticos de enfermagem identificados. Destaca-se que o " $n$ " apresentado pode ser diferente tanto para os DE, quanto para as características definidoras (CD) e fatores relacionados (FR), visto que cada participante pode ou não apresentar o conjunto completo de DE, CD e FR.

Figura 2. Distribuição da frequência das características definidoras e fatores relacionados segundo os diagnósticos de enfermagem. Redenção, 2019.

\begin{tabular}{|c|c|c|}
\hline DOMINIO 2. NUTRIÇÃO & $\mathbf{n}$ & $\%$ \\
\hline DIAGNÓSTICO "AMAMENTAÇÃO INEFICAZ" & 13 & 28,8 \\
\hline \multicolumn{3}{|l|}{ CARACTERISTICAS DEFINIDORAS } \\
\hline Ausência de produção de leite com estimulação do mamilo & 2 & 4,4 \\
\hline Sinais insuficientes de liberação de ocitocina & 1 & 2,2 \\
\hline Sucção na mama não sustentada & 2 & 4,4 \\
\hline Suprimento de leite inadequado percebido & 5 & 11,1 \\
\hline Esvaziamento insuficiente de cada mama por amamentação & 3 & 6,6 \\
\hline \multicolumn{3}{|l|}{ FATORES RELACIONADOS } \\
\hline Suprimento de leite inadequado & 3 & 6,6 \\
\hline Reflexo de sucção do lactente insatisfatório & 1 & 2,2 \\
\hline Atraso no estágio II da lactogenese & 5 & 11,1 \\
\hline Oportunidade insuficiente de sugar a mama & 3 & 6,6 \\
\hline Dor materna & 1 & 2,2 \\
\hline DOMINIO4. ATIVIDADE/REPOUSO & n & $\%$ \\
\hline DIAGNÓSTICO "RISCO DE DÉBITO CARDÍACO DIMINUÍDO" & 2 & 4,4 \\
\hline DIAGNÓSTICO "PADRÃO RESPIRATÓRIO INEFICAZ" & 8 & 17,7 \\
\hline \multicolumn{3}{|l|}{ CARACTERISTICAS DEFINIDORAS } \\
\hline Taquipneia & 7 & 15,5 \\
\hline Uso da musculatura acessória para respirar & 1 & 2,2 \\
\hline \multicolumn{3}{|l|}{ FATORES RELACIONADOS } \\
\hline Alteração na frequência cardíaca (CONDIÇÃO ASSOCIADA) & 2 & 4,4 \\
\hline Hiperventilação & 7 & 15,5 \\
\hline Fadiga & 1 & 2,2 \\
\hline DOMINIO 11. SEGURANÇA/PROTEÇÃO & $\mathbf{n}$ & $\%$ \\
\hline
\end{tabular}


Research, Society and Development, v. 9, n. 8, e688986058, 2020

(CC BY 4.0) | ISSN 2525-3409 | DOI: http://dx.doi.org/10.33448/rsd-v9i8.6058

\begin{tabular}{|c|c|c|}
\hline DIAGNÓSTICO "RISCO DE INFECÇÃO" & $\mathbf{3 6}$ & $\mathbf{8 0}$ \\
\hline FATOR DE RISCO & 35 & 77,7 \\
\hline Vacinação inadequada & 1 & 2,2 \\
\hline Alteração na integridade da pele & $\mathbf{n}$ & $\mathbf{\%}$ \\
\hline DIAGNÓSTICO "RISCO DE DESENVOLVIMENTO ATRASADO" & $\mathbf{2 5}$ & $\mathbf{5 5 . 5}$ \\
\hline FATORES DE RISCO & 2 & 4,4 \\
\hline Cuidado pré-natal tardio & 3 & 6,6 \\
\hline Cuidado pré-natal insuficiente & 13 & 28,8 \\
\hline Desfavorecido economicamente & 1 & 2,2 \\
\hline Idade materna $\leq 15$ anos & 6 & 13,3 \\
\hline Idade materna $\geq 35$ anos & & \\
\hline
\end{tabular}

Fonte: Dados da pesquisa (2019).

Conforme estabelecido no Figura 2, no DE Amamentação ineficaz, a característica definidora (CD) mais prevalente foi Suprimento de leite inadequado percebido $5(11.1 \%)$ e o fator relacionado (FR) foi Atraso no estágio II da lactogenese 5 (11.1\%). O DE Débito cardíaco diminuído foi identificado em dois participantes, a qual teve como CD bradicardia 2 (4.4\%) e condição associada, alteração na frequência cardíaca 2 (4.4\%). O DE Padrão respiratório ineficaz teve $\mathrm{CD}$ mais frequente taquipneia $7(15.5 \%)$ e FR hiperventilação 7(15.5\%). O DE Risco de infecção ocorreu em 36 participantes que corresponde à 80\% da amostra com o fator de risco vacinação inadequada $35(77.7 \%)$ e alteração na integridade da pele $1(2.2 \%)$. Vale ressaltar que o fator de risco vacinação inadequada foi registrado porque as crianças da maternidade do estudo não eram vacinadas ao nascimento com as vacinas BCG e anti-Hepatite B; as mães eram orientadas a procurar uma Unidade de Atenção Primária à Saúde, quando tivessem alta hospitalar, para vacinar seus filhos. O DE Risco de desenvolvimento atrasado, o fator de risco mais prevalente foi desfavorecido economicamente $13(28.8 \%)$. 


\section{Discussão}

Quanto às características ao nascimento do bebê, estas foram importantes para subsidiar a identificação dos diagnósticos de enfermagem, podendo algumas delas serem consideradas características definidoras para os diagnósticos.

No que se refere aos dados sócio demográficos das mães, estes foram importantes para justificar a presença de possíveis diagnósticos, podendo se configurar como fatores relacionados ou fatores de risco para a resposta humana identificada.

Como exemplo do que foi mencionado anteriormente, tem-se a idade materna. Resultados obtidos no estudo de Silva (2017) sugerem que a gravidez no extremo da vida reprodutiva encontra-se associado ao acompanhamento pré-natal menos eficiente, à maior incidência de parto cesáreo, nascimento pré-termo e baixo peso ao nascer. No presente estudo, apesar da maioria das mães estarem em uma faixa etária não considerada de risco, 16 mães tinham idades menor que 16 anos ou maior que 35 anos. Estudos apontam que a idade materna avançada, por exemplo, tem implicações na qualidade de saúde dos filhos, como o estudo de Nakadonari \& Soares (2006), que apontou que a probabilidade de ocorrência de trissomia do cromossomo 21 aumenta significativamente depois que a mãe completa 35 anos".

Outros dados das mães, apresentados neste estudo, também podem levar a ocorrência de características ou alterações nos bebês ao nascimento, e que podem perdurar por muito tempo: local de residência, pois as mães que não são do município tiveram que se deslocar até lá para parir e as condições as quais ocorreu esse deslocamento podem trazer prejuízos ao bebê; entidade familiar, escolaridade, ocupação e renda são fatores que podem ter influenciado todo o pré-natal (realização das consultas, conhecimento prévio, conhecimento adquirido, alimentação e cuidados) dessas mães e, consequentemente, o estado de nascimento desses bebês.

Desta forma, a partir dos dados mencionados anteriormente, dos bebês ao nascimento e das mães, foram identificados cinco diagnósticos de enfermagem (DE) em quatro domínios distintos da Taxonomia II da NANDA-I (NANDA, 2018).

No domínio 2 Nutrição, classe 1- ingestão, foi encontrado em $28.8 \%$ da amostra o DE Amamentação ineficaz. Esse diagnóstico também foi identificado no estudo de Oliveira (2013) sobre diagnósticos de enfermagem em recém-nascidos com alteração glicêmica, em $26.66 \%$ da amostra; percentual bem similar ao do presente estudo. Ressalta-se, no entanto, que esse estudo mencionado foi realizado com recém-nascidos com uma alteração específica, 
que pode ter influenciado a existência do diagnóstico. No entanto, um outro estudo (Silva, Alves, Macedo, Bezerra et al 2013) que teve como objetivo identificar, em uma unidade de Alojamento Conjunto, diagnósticos de enfermagem relacionados ao fenômeno da também evidenciou o mesmo DE em $13.3 \%$ da amostra de seu estudo. Nesse último tanto o ambiente como a população foi similar ao do presente estudo.

A identificação desse diagnóstico se faz importante para que ações sejam planejadas para que o bebê não tenha sua nutrição prejudicada, além de outros prejuízos. Segundo Azevedo, Caminha, Cruz, Silva et al (2019), a amamentação traz um conjunto de efeitos benéficos na saúde da criança e no seu desenvolvimento. Os profissionais de saúde, incluindo o enfermeiro precisa estar preparado para promover o aleitamento materno, sempre levando em consideração os aspectos emocionais e físicos da mulher e os benefícios que esse aleitamento trará para mãe e bebê.

Um aspecto que pode influenciar diretamente a amamentação é evidenciado nos estudos de Freitas, Melo, Pereira et al (2014) e Silva et al (2013), que é a escolaridade das mães. Esses autores referem que ter poucos anos de estudos pode desfavorecer a continuidade da amamentação. Ao contrário, mulheres com mais anos de estudo tende a amamentar seus filhos por tempo mais prolongado.

No presente estudo, apenas $40 \%$ das mães tem o ensino médio completo e $15,5 \%$ tem o nível superior, fato que pode justificar ou influenciar a presença do diagnóstico amamentação ineficaz em $28.8 \%$ da amostra.

Desta forma, a sistematização de assistência de enfermagem torna-se útil, pois irá direcionar o cuidado de enfermagem ao binômio mãe-filho, dando toda a assistência necessária para o processo de amamentação.

No domínio 4 Atividade Repouso, foram encontrados dois DE, Padrão respiratório ineficaz (17.7\%) e Débito cardíaco diminuído (4.4\%).

O DE Padrão respiratório ineficaz é definido "como inspiração e/ou expiração que não proporciona ventilação adequada". Este diagnóstico mantém relação com a mecânica ventilatória prejudicada do RN (Lucena, 2017). Neste estudo, foi representado pelas características definidoras: uso da musculatura acessória para respirar e taquipneia. Esse mesmo diagnóstico foi encontrado nos estudos de D'Angelo, Darli, Leite, Furtado et al (2010), que evidenciou uma frequência de $16.1 \%$ em prematuros sob cuidados intermediários, e de Oliveira (2013) com uma prevalência de 36.6\%. Estes achados corroboram com os dados verificados neste estudo. No entanto, nos dois estudos mencionados, os recém-nascidos apresentavam alterações específicas de saúde. 
Lopes, Monteiro \& Oliveira, et al (2017) sublinham que se faz necessário ponderar que essa resposta humana pode se relacionar com as doenças do sistema respiratório, causa importante de internação pediátrica no Brasil. Portanto, justifica-se a importância e necessidade da identificação deste diagnóstico o mais precoce possível. É de responsabilidade da enfermagem prestar cuidados ao RN que assegurem uma boa transição da vida intrauterina para extrauterina e previnam agravos que possam acometê-lo. Assim, a monitoração da mecânica pulmonar é um dos cuidados que merece atenção e habilidade por parte do enfermeiro.

O outro diagnóstico identificado do mesmo domínio, Risco de débito cardíaco diminuído, é definido pela NANDA-I (2018) como suscetibilidade a volume de sangue bombeado pelo coração inadequado para atender às demandas metabólicas do organismo que pode comprometer a saúde. Neste estudo, ele esteve presente em $4.4 \%$, com a condição associada, alteração na frequência cardíaca.

Após o parto, ocorrem adaptações fisiológicas para que o neonato assuma suas funções vitais, que envolvem modificações funcionais complexas de todos os sistemas do organismo incluindo o sistema cardiovascular. Neste aspecto, os profissionais devem estão capacitados para reconhecer situações que requeiram cuidados de emergência, devido às chances de distúrbios e complicações (Bastos, 2010).

No domínio 11, Segurança e proteção, foi identificado o DE Risco de infecção, presente em $80 \%$ dos bebês, sendo a característica definidora mais frequente vacinação inadequada (77.7\%). Este foi o diagnóstico mais presente neste estudo.

O risco de infecção é definido como suscetibilidade à invasão e multiplicação de organismos patogênicos que pode comprometer a saúde (NANDA, 2018). D’Angelo et al (2010) referem que os neonatos são susceptíveis à infecção, pois saíram do meio estéril (útero materno) para um ambiente contaminado. Segundo a Sociedade Brasileira da Imunização (SBIM, 2017), ter a vacinação em dia diminui as internações hospitalares e o risco de adoecimento. No contexto de nascimento, e antes da alta hospitalar, nas instituições hospitalares públicas, os bebês devem ser vacinados com as vacinas BCG e anti-Hepatite B.

Para este mesmo diagnóstico, outra característica definidora presente foi integridade da pele prejudicada, em 2,2\% da amostra. Mesmo sendo em pequena porcentagem, este aspecto merece muita atenção, pois os cuidados com a pele do RN devem fazer parte da assistência de enfermagem.

No domínio 13, Crescimento e Desenvolvimento, foi identificado o DE Risco de desenvolvimento atrasado em $55.5 \%$ dos bebês. A CD mais prevalente foi desfavorecimento 
econômico $(28.8$ \%). Ressalta-se que os fatores genéticos, ambientais e sociais têm uma influência direta na saúde das pessoas.

O estudo apresentou limitação, pois os dados foram coletados em uma única instituição do Estado, mesmo sendo referência para uma região específica. Apesar da limitação, consideramos o estudo pertinente por abordar um tema atual, taxonomia dos diagnósticos de enfermagem e recém-nascidos a termo, ancorando seus resultados a contribuições para a profissão de enfermagem e para uma região do Estado na qual os profissionais de enfermagem não utilizam a Sistematização de Enfermagem em sua prática diária.

\section{Considerações Finais}

A partir do estudo, foi possível identificar cinco diagnósticos de enfermagem com suas características definidoras, fatores relacionados ou fatores de risco em recém-nascidos de uma maternidade do interior do Estado do Ceará. Mesmo que a sistematização da assistência de enfermagem ainda não seja uma prática instituída no local do estudo, esse é um aspecto que, legalmente, deve ser incorporado, e a identificação de diagnósticos de enfermagem é o primeiro passo. Desta forma, estudos como este podem contribuir na implementação da sistematização, além de ajudar os profissionais a organizar o cuidado prestado a esse público.

Ressalta-se, ainda, a importância do pensamento crítico e reflexivo, baseado em evidências científicas por parte do enfermeiro para assegurar uma assistência qualificada.

Neste âmbito, este estudo contribuiu para embasar a prática de enfermagem e também serve como fonte documental para futuras pesquisas, como a validação dos diagnósticos como também de despertar para o estudo dos resultados e intervenções de enfermagem que também integram a sistematização da assistência de enfermagem.

Considera-se também que os achados abrolham a realização de mais estudos com esta temática, para melhor compreensão e conhecimentos sobre os diagnósticos de enfermagem em recém-nascidos, podendo subsidiar a escolha de melhores resultados a serem alcançados e intervenções a serem implementadas.

\section{Agradecimentos}

Agradecemos a direção do hospital e maternidade Paulo Sarasate. Agradecemos, também, todos os profissionais de enfermagem e as puérperas que colaboraram para a coleta de dados. 


\section{Referências}

Azevedo, P. T. Á. C. C., Caminha, M. F. C., Cruz, R. S. B. L. C., Silva, S. L., Paula, W. K. A. S., \& Batista Filho, M.. (2019). Estado nutricional de crianças em amamentação exclusiva prolongada no Estado de Pernambuco. Revista Brasileira de Epidemiologia, 22, e190007. Epub March 14, 2019.https://doi.org/10.1590/1980-549720190007

Bastos, L. M. (2010). Sistematização da assistência de enfermagem ao recém-nascido por meio da educação permanente em saúde. Fortaleza.

Del'Angelo, N., Góes, F. S. N., Dalri, M. C. B., Leite, A. M., Furtado, M. C. C., \& Scochi, C. G. S.. (2010). Diagnósticos de enfermagem de prematuros sob cuidados intermediários. Revista Brasileira de Enfermagem, 63(5), 755-761. https://doi.org/10.1590/S0034$\underline{71672010000500010}$

Elizalde, A. C., \& Almeida, M. A. (2006). Percepções de enfermeiras de um hospital universitário sobre a implantação dos diagnósticos de enfermagem. Revista Gaúcha de Enfermagem, 27(4). 564-74.

Freitas, L. J. Q., Melo, N. C. C. C., Pereira, M. M. Q., Valente, M. M. P., Moura, E. R. F., Américo, C. F., \& Sousa, C. S. P. (2014). Amamentação ineficaz entre nutrizes atendidas em unidades básicas de saúde. Revista Enfermagem UERJ, Rio de Janeiro, 22(1), 103-10.

Garcia, T. R., \& Nóbrega, M. M. L. (2004). Contribuição das teorias de enfermagem para a construção do conhecimento da área. Revista Brasileira Enfermagem, 57(2), 228-32.

Gordon, M. (1994). Nursing diagnosis: process and application. 3ed. St. Louis: Mosby.

Kenner, C. (2001). Adaptação do Neonato. In: Enfermagem Neonatal. 2. ed. Rio de Janeiro: Reichmsnn \& Affonso. Capítulo. 1, 26-27.

Leon, P. A. P., \& Nóbrega, M. M. L. (2012). Diagnóstico de enfermagem em crianças hospitalizadas utilizando a NANDA-I: estudo de caso. Online Brazilian Journal of Nursing; $11(1), 68-78$. 
Lopes, T. A. M. C., Monteiro, M. de F. V., Oliveira, J. D. de., Oliveira, D. R. de., Pinheiro, A. K. B. \& Damasceno, S. S. et al (2017). Diagnósticos de enfermagem em crianças hospitalizadas. Revista Rene; 18(6). 756-62.

Lucena, L. F., Reis, A. T., Araújo, B.. B. M., Pacheco, S. T. A. \& Vieira, R. de. F. (2017). Diagnósticos e intervenções de enfermagem ao neonato com Síndrome de Donohue (Leprechaunismo): estudo de caso [Nursing diagnoses andinterventions for a neonatewithDonohueSyndrome (Leprechaunism): a case study]. Revista Enfermagem UERJ, [S.1.], 25, e20387. DOI: https://doi.org/10.12957/reuerj.2017.20387.

Monteiro, F. P. M. (2013). Construção de um diagnóstico de enfermagem na categoria promoção da saúde para o crescimento/desenvolvimento: estudo em lactentes. (Tese de Doutorado), Universidade Federal do Ceará. Faculdade de Farmácia, Odontologia Enfermagem. Programa de Pós-Graduação em Enfermagem. Fortaleza.

NANDA Internacional. (2010). Diagnósticos de enfermagem da NANDA: definições $e$ classificação 2009- 2010. Porto Alegre: Artmed.

NANDA Internacional. (2018). Diagnósticos de Enfermagem da NANDA I: definições e classificação 2018-2020. Porto Alegre: Artmed.

Nakadonari, E. K., Soares, A. A. (2006). Síndrome de Down: considerações gerais sobre a influência da idade materna avançada. Arquivo Mudi. 10(2), 5-9.

Oliveira, S. I. M., Souza, N. L., Costa \& Silva, R. K. (2013). Diagnósticos de enfermagem em recém-nascido com alterações glicêmicas. Revista Cogitare Enfermagem, [S.1.], 18(4). DOI: http://dx.doi.org/10.5380/ce.v18i4.34923.

Primo, M. G. B., et al. (2010). Adesão à prática de higienização das mãos por profissionais de saúde de um Hospital Universitário. Revista Eletrônica de Enfermagem [Internet], 12(2), p. 266-71.

Silva, E. P., Alves, A. R., Macedo, A. R. M., Bezerra, R. M. S. B., Almeida, P. C., \& Chaves, E. M. C.. (2013). Diagnósticos de enfermagem relacionados à amamentação em unidade de 
alojamento conjunto. Revista Brasileira de Enfermagem, 66(2), 190-195. https://doi.org/10.1590/S0034-71672013000200006

Sociedade Brasileira de Imunizações. (2017). Imunização: tudo o que você sempre quis saber. Silva, P. C. (2017). Influencias da idade materna sobre os resultados perinatais de nascidos vivos de São Luís/maranhão. Universidade Federal de Maranhão.

Souza, S. E. M., Ferreira, M. J. M., Chaves, E. M. C., Barbora, R. C. M., \& Dodt, R. C. M. (2012). Diagnósticos de enfermagem em recém-nascidos internados em unidade de terapia intensiva neonatal: uma pesquisa bibliográfica. Revista Diálogos Acadêmicos, Fortaleza, 2(1).

\section{Porcentagem de contribuição de cada autor no manuscrito}

Fatumata Binta Embalo - 23\%

Emília Soares Chaves Rouberte - 23\%

Isaura Leticia Tavares Palmeira Rolim - 14\%

Rafaella Pessoa Moreira - 10\%

Maria Isis Feire de Aguiar-10\%

Débora Oliveira Pordeus - $10 \%$

Jocelha Maria Costa Almeida - 10\% 